\title{
Validation of an extremely patient-friendly progestin- primed ovarian stimulation protocol in normal and high responders by comparing with conventional antagonist protocol: a retrospective study
}

Chen-Yu Huang

Institute of Clinical Medicine, National Yang-Ming University

Guan-Yeu Chen

Taipei Veterans General Hospital

Miawh-Lirng Shieh

Taipei Veterans General Hospital

Hsin-Yang Li ( $\sim$ lihy@vghtpe.gov.tw)

Taipei Veterans General Hospital

Research

Keywords: Progestin-primed ovarian stimulation (PPOS); long-acting FSH; patient-friendly; antagonist

Posted Date: April 29th, 2020

DOI: https://doi.org/10.21203/rs.3.rs-25012/v1

License: (c) (1) This work is licensed under a Creative Commons Attribution 4.0 International License.

Read Full License 


\section{Abstract}

Background. Our previous study has shown a satisfactory reproductive outcome resulting from the extremely patient-friendly ovarian stimulation protocol of long-acting follicle stimulation hormone (FSH) plus oral medroxyprogesterone acetate (MPA). The present study was aimed to compare the efficacy of the extremely patient-friendly ovarian stimulation protocol with that of antagonist protocol.

Methods. A retrospective study was conducted on normal and high responders aged from 24 to 39 years starting ovarian stimulation for IVF/ICSI with long-acting FSH at early follicular phase in a tertiary fertility center in Taiwan between December 2016 and August 2017. To prevent premature luteinizing hormone (LH) surge, oral MPA was applied in some cycles (group 1), whereas antagonist protocol was applied in other cycles (group 2). Freezing all embryos was applied to all patients in both groups. Duration of stimulation, number of shots and visits before trigger, incidence of premature LH surge, number of oocytes retrieved, fertilization rate, cleavage rate, rate of good embryos available, incidence of ovarian hyperstimulation syndrome, cumulative clinical pregnancy rate, and cumulative live birth rate per retrieval were compared between groups.

Results. One hundred and ten cycles of IVF/ICSI were included, among which there were fifty-seven cycles in group 1 and fifty-three cycles in group 2. Starting with long-acting FSH, group 1 (MPA) required much fewer injections and visits but had comparable reproductive outcomes to those in group 2 (antagonist protocol).

Conclusions. Long-acting FSH plus MPA use for ovarian stimulation is extremely friendly for the patients and demonstrates satisfactory stimulation and reproductive outcomes that are comparable to those of antagonist protocol.

\section{Introduction}

Conventional ovarian stimulation in assisted reproductive technology (ART) burdens patients with plenty of shots, including daily gonadotrophins for follicle development and gonadotrophin-releasing hormone $(\mathrm{GnRH})$ analogues for premature luteinizing hormone $(\mathrm{LH})$ surge prevention. In the past, GnRH agonist was the most popular way to prevent LH elevation, which was named long protocol owing to its lengthy course of daily injections [1]. Over the last decade, it was gradually replaced by GnRH antagonist protocol, which is characterized by immediate suppression of LH with fewer injections and lower risk of ovarian hyperstimulation syndrome (OHSS) [2]. Emerging from the demand for urgent fertility preservation in cancer patients [3], luteal phase stimulation was adopted [4] in conjunction with freeze-all strategy, wherein the competence of the oocytes/embryos as well as the pregnancy and neonatal outcomes have already been validated on a large scale [5-7]. Stemming from the awareness of endogenous progesterone's LH suppressive capability, oral progestogen was applied and proven to be an effective alternative for $\mathrm{LH}$ rise prevention, namely progestin-primed ovarian stimulation (PPOS) [8-10]. Thus, multiple injections of $\mathrm{GnRH}$ analogues can be omitted. 
Previous studies regarding PPOS, however, all utilized daily injection of gonadotrophins such as human menopausal gonadotrophin (HMG), and most of them compared PPOS with short agonist protocol [8, 9, 11-13]. To pursue a more patient-friendly way of ovarian stimulation in ART cycles, we combined PPOS with long-acting follicle stimulation hormone (FSH), corifollitropin alfa, and demonstrated a competent reproductive outcome in normal/high responders with cumulative ongoing pregnancy rate per oocyte retrieval of 53.1\%. In the aforementioned PPOS using long-acting FSH, only 3.6 injections shots and 1.4 visits were needed on average before trigger [14]. According to a worldwide survey of ART practices, more than three-quarters of cycles utilized an antagonist protocol [15]; however, researches seldom focused on the comparison between PPOS and antagonist protocol. The aim of our present study is to compare the reproductive outcomes of long-acting FSH (corifollitropin alfa) use in PPOS versus in antagonist protocol.

\section{Materials And Methods}

\section{Study setting and patients}

A retrospective study was performed at the Center for Reproductive Medicine in Taipei Veterans General Hospital, and undertaken by means of chart review. All patients were counseled and informed consents for the stimulation protocol and related procedures were provided by infertility specialists. From December 2016 to August 2017, 235 cycles of ovarian stimulation for ART commenced with long-acting FSH (corifollitropin alfa) at early follicular phase, which was defined as either the first five days of menstruation cycle or basal serum estradiol less than $75 \mathrm{pg} / \mathrm{mL}$ along with basal serum progesterone below $1 \mathrm{ng} / \mathrm{mL}$. Among these cycles, patients with one of the following conditions were excluded from the study: (i) age over forty years, (ii) antral follicle count (AFC) below 7, (iii) basal FSH more than $10 \mathrm{IU} / \mathrm{L}$, (iv) a previous poor ovarian response ( $\leq 3$ oocytes retrieved with a conventional stimulation protocol), ( $v$ ) body mass index (BMI) above 30, (vi) hypogonadotropic hypogonadism, and (vii) uterine abnormalities. Ethical approval for this study was obtained from the Institutional Review Board of Taipei Veterans General Hospital (2018-12-005BC)

\section{Treatment protocol of ovarian stimulation and oocyte retrieval}

Transvaginal ultrasound and serum hormone measurements (FSH, LH, estradiol [E2], and progesterone $[\mathrm{P}]$ ) were performed on the starting day just before long-acting FSH (corifollitropin alfa) injection, of which the dosage was determined by the patient's body weight (150 micrograms for $>60 \mathrm{~kg}$ and 100 micrograms for $\leq 60 \mathrm{~kg}[16]$ ). In cycles with PPOS (PPOS protocol, group 1), patients took medroxyprogesterone acetate (MPA) $5 \mathrm{mg}$ BID from the day after long-acting FSH injection, which was published elsewhere [14]. In cycles with antagonist protocol (antagonist protocol, group 2), daily cetrorelix $0.25 \mathrm{mg}$ subcutaneous (sc) injection was initiated from the evening of stimulation day 5 . Seven days after long-acting FSH injection, the folliculogenesis was monitored by transvaginal ultrasound along with serum hormone levels of E2, LH and P. As long as at least three leading follicles reached above $17 \mathrm{~mm}$ in diameter, patients were triggered at night. If the follicle development was not adequate for trigger, 
additional HMG 150-225 IU/day would be given for days depending on the prediction according to the monitoring on stimulation day 8 . If necessary, additional follicle monitoring would be performed every 23 days to evaluate whether the criterion for trigger was met. Patients in group 1 took the final tablet of MPA in the morning of the trigger day, whereas patients in group 2 received the final cetrorelix injection in the evening before the trigger day. Triggering was given by subcutaneous injection of triptorelin $0.2 \mathrm{mg}$ with or without human chorionic gonadotrophin (hCG) 1500-6500 IU, depending on the risk evaluation for early onset OHSS. A simplified illustration of the two treatment protocols is shown in Fig. 1. Ovum pick-up was performed 34-38 $\mathrm{h}$ after triggering, followed by in vitro fertilization (IVF) and/or intracytoplasmic sperm injection (ICSI) according to the conditions of the sperm. All embryos were vitrified at pronuclear stage, or on day 2 , or on day 3 , or on day 5 after oocyte retrieval depending on the number of fertilized oocytes available.

\section{Endometrial preparation for frozen-thawed embryo transfer}

In frozen-thawed embryo transfer (FET) cycles, oral estradiol valerate $6 \mathrm{mg}$ twice a day was administered from menstrual cycle day 2-4 onwards. After oral estradiol valerate had been taken for 1014 days, the thickness of endometrium was measured by transvaginal sonography. Additionally, serum hormone tests (E2, LH, P) were performed to confirm that no spontaneous follicle growth and ovulation occurred. Once the endometrial thickness reached more than $7 \mathrm{~mm}$, oral estradiol valerate supplement was continued and the patient began to use vaginal micronized progesterone gel $90 \mathrm{mg}$ twice a day and vaginal micronized progesterone soft capsules $400 \mathrm{mg}$ every night until either ten weeks of gestation or confirmed failure of pregnancy. The timing of thawing and transfer of frozen embryos was determined based on the stage they were vitrified, and synchronized with the duration of progesterone exposure of the endometrium. Embryos frozen at the pronuclear stage were warmed after one day of vaginal progesterone treatment, followed by transfer one or two days later according to the number of viable embryos. Embryos frozen on post-retrieval day 2 or day 3 or day 5 were thawed after 2 or 3 or 5 days of luteal support, respectively, and were transferred on the same day. Serum $\beta$-hCG level was checked after two weeks of luteal support. If the serum $\beta$-hCG level was above $10 \mathrm{IU} / \mathrm{L}$, transvaginal sonography was done three weeks later to detect the site of gestational sacs along with the fetal heartbeat.

\section{Outcome measures}

Demographic variables recorded for each patient included age, BMI, AFC, serum levels of basal FSH and LH, primary infertility, and the indication for IVF/ICSI treatments. Parameters about ovarian stimulation, oocytes, embryos, and pregnancy outcomes after FET were listed below: duration of stimulation, the number of injections and visits before trigger, premature LH surge, the number of oocytes retrieved, fertilization rate, cleavage rate, the rate of good embryos available, OHSS, cumulative clinical pregnancy rate, and cumulative live birth rate per retrieval. In the study, peak E2 and LH levels on the trigger day were not necessarily measured due to freeze-all policy. A serum LH concentration over $10 \mathrm{IU} / \mathrm{L}$ or rising above twice the basal value before trigger was regarded as premature LH surge. Embryos growing to 2-4 cells on one day after pronuclear stage with grade I or II morphology [17] were considered as good embryos. Cumulative clinical pregnancy rate was counted as pregnancies above 7 weeks with intrauterine fetal 
heart beat detected divided by the number of retrieval cycles whose embryos were all transferred or confirmation of intrauterine pregnancies with active fetal heartbeat. Live birth was defined as delivery above 32 weeks of gestational age.

\section{Statistical Analysis}

Chi-square test was used for comparisons of nominal variables between the two groups. Continuous parameters were analyzed with student's t- test, presented as the mean \pm standard deviation (SD). The SPSS statistical package (version 24; SPSS Inc., Chicago, IL, USA) was used for analysis, and a p value of less than 0.05 was considered to be statistically significant.

\section{Results}

In the study period, a total of 110 cycles of IVF/ICSI stimulated with corifollitropin alfa were included in this retrospective study. Among the 110 cycles, there were fifty-seven cycles of PPOS (group 1) and fiftythree cycles of antagonist protocol (group 2).

In regard to demographic data, age, BMI, AFC, serum basal FSH and LH levels, primary infertility, and the indication for IVF/ICSI treatments were comparable between two groups. (Table 1) 
Table 1

Demographic characteristics

\begin{tabular}{|c|c|c|c|}
\hline & $\begin{array}{l}\text { long-acting FSH+ } \\
\text { MPA } \\
\text { (group } 1, n=57 \text { ) }\end{array}$ & $\begin{array}{l}\text { long-acting FSH + } \\
\text { Cetrorelix } \\
\text { (group 2, n= 53) }\end{array}$ & $\begin{array}{l}P \text { - } \\
\text { Value }\end{array}$ \\
\hline Age (Mean $\pm S D)$ & $34.8 \pm 2.73$ & $34.9 \pm 3.41$ & 0.865 \\
\hline BMI (Mean \pm SD) & $21.54 \pm 2.55$ & $21.75 \pm 2.97$ & 0.693 \\
\hline $\mathrm{BMI} \leq 24(\mathrm{n}, \%)$ & $45,78.95 \%$ & $39,73.58 \%$ & \multirow[t]{2}{*}{0.519} \\
\hline $\mathrm{BMI}>24(\mathrm{n}, \%)$ & $12,21.05 \%$ & $14,26.42 \%$ & \\
\hline AFC (Mean \pm SD) & $18.8 \pm 10.82$ & $16.8 \pm 10.61$ & 0.318 \\
\hline Basal FSH (IU/L) (Mean \pm SD) & $6.37 \pm 1.15$ & $6.48 \pm 1.92$ & 0.733 \\
\hline Basal LH (IU/L) (Mean \pm SD) & $4.37 \pm 2.29$ & $3.90 \pm 1.71$ & 0.235 \\
\hline Primary Infertility (n, \%) & $35,61.40 \%$ & $26,49.06 \%$ & 0.193 \\
\hline The indication for IVF/ICSI & $20,35.08 \%$ & $16,30.19 \%$ & 0.283 \\
\hline Male Factor (n, \%) & $9,15.78 \%$ & $15,28.30 \%$ & \\
\hline Tubal Factor (n, \%) & \multirow[t]{2}{*}{$28,49.12 \%$} & \multirow[t]{2}{*}{$22,41.51 \%$} & \\
\hline Other $(\mathrm{n}, \%)$ & & & \\
\hline
\end{tabular}

There was no significant difference in the duration of stimulation, but the numbers of injections and visits before trigger were significantly lower in group 1 . The number of oocytes retrieved, fertilization rate, cleavage rate, and good embryo rate on day 2 showed no significant difference between two groups. None of the patients developed either premature LH surge or OHSS in either group. (Table 2) 
Table 2

Stimulation characteristics and data of oocytes and embryos

\begin{tabular}{|llll|}
\hline & $\begin{array}{l}\text { long-acting FSH + MPA } \\
\text { (group 1, } \mathrm{n}=57) \\
\text { Mean } \pm \text { SD }\end{array}$ & $\begin{array}{l}\text { long-acting FSH + Cetrorelix } \\
\text { (group 2, } \mathrm{n}=53 \text { ) } \\
\text { Mean } \pm \text { SD }\end{array}$ & P-Value \\
\hline No. of injection before trigger & $3.5 \pm 1.24$ & $9.7 \pm 2.89$ & $<0.001^{*}$ \\
\hline No. of visits between & $1.4 \pm 0.49$ & $1.9 \pm 0.56$ & $<0.001^{*}$ \\
\hline long-acting FSH shot and trigger & & & 0.415 \\
\hline Duration of stimulation & $9.4 \pm 1.32$ & $9.2 \pm 1.22$ & - \\
\hline Premature LH surge & 0 & 0 & 0.908 \\
\hline No. of oocytes retrieved & $13.9 \pm 7.32$ & $13.7 \pm 8.57$ & 0.059 \\
\hline Fertilization rate & $77.93 \% \pm 17.53 \%$ & $70.35 \pm 23.64 \%$ & 0.131 \\
\hline Cleavage rate & $90.78 \% \pm 10.94 \%$ & $86.91 \% \pm 14.66 \%$ & 0.909 \\
\hline D2 good embryo rate & $63.26 \% \pm 23.71 \%$ & $62.67 \% \pm 27.88 \%$ & - \\
\hline Incidence of OHSS & 0 & 0 & \\
\hline
\end{tabular}

Till submission of this work, 54 patients in group 1 and 48 patients in group 2 have either transferred all their cryopreserved embryos without pregnancy or achieved intrauterine pregnancy with good fetal heartbeat. Thirty of these 54 patients in group 1 and twenty-eight of these 48 patients in group 2 conceived through FET showing good fetal heartbeat at 7 weeks of gestation. Therefore, the cumulative clinical pregnancy rate per ovum pickup was $55.56 \%$ in group 1 and $58.33 \%$ in group 2, between which there was no statistically significant difference. At the time of submission, there were 28 patients in group 1 and 26 patients in group 2 having live birth without major problems, which showed no significant difference in percentage between groups (Table 3). Regarding the hormonal change in patients undergoing PPOS (group 1) versus antagonist protocol (group 2), the serum E2, LH, and P levels at basal status and at trigger were presented with mean and SD in Fig. 2. Due to freeze-all strategy, serum hormone tests on the trigger day were not necessarily performed; therefore, the data in Fig. 2 came from only 14 patients for each group respectively. 
Table 3

Pregnancy outcomes of frozen-thawed embryo transfer (FET)

\begin{tabular}{|lllr|}
\hline & $\begin{array}{l}\text { long-acting FSH } \\
\text { MPA } \\
\text { (group 1) }\end{array}$ & $\begin{array}{l}\text { long-acting FSH+ } \\
\text { Cetrorelix } \\
\text { (group 2) }\end{array}$ & $\begin{array}{l}\text { P- } \\
\text { Value }\end{array}$ \\
\hline $\begin{array}{l}\text { Cumulative clinical pregnancy rate with } \\
\text { positive FHB }\end{array}$ & $55.56 \%(30 / 54)$ & $58.33 \%(28 / 48)$ & 0.779 \\
$\begin{array}{l}\text { Cumulative live birth rate at the time of } \\
\text { submission }\end{array}$ & $51.85 \%(28 / 54)$ & $54.17 \%(26 / 48)$ & 0.816 \\
\hline
\end{tabular}

\section{Discussion}

The significance of our regimen is that one shot of long-acting FSH substitutes for seven daily injections and oral MPA co-administration replaces many shots of GnRH analogue, which decreases psycho/physical burden and time-exhausting visits along with more convenience and less cost (MPA versus GnRH antagonist). To our knowledge, most publications about PPOS to date were based on HMG, and whether MPA-PPOS was comparable with antagonist protocol in long-acting FSH application has not been reported. Thus, our study is the first one comparing PPOS versus antagonist protocol with longacting FSH use.

Thanks to recombinant DNA technologies, one single shot of corifollitropin alfa, wherein the FSH $\beta$ subunit is extended by a carboxy-terminal peptide of the hCG $\beta$-subunit [18], can support folliclestimulating activity equivalent to the circulating FSH level sufficient for multiple follicle growth throughout the first week [19] and thus replace seven daily FSH injections with compatible reproductive outcomes in conventional protocols [20]. There is no exogenous LH-effect agent given during the first week of stimulation. The major uniqueness of our protocol compared with other PPOS regimens, accordingly, is to rely on the remaining endogenous LH activity under progestin suppression in the first 7 days of ovarian stimulation. Debate about the role of LH in ovarian stimulation is still continuing. Due to diverse results, the necessity of LH supplementation for patients undergoing IVF/ICSI cycles is still uncertain [21,22]. The updated Cochrane review showed no clear evidence of a difference between rLH combined with $\mathrm{rFSH}$ and $\mathrm{rFSH}$ alone in live birth rates in spite of more ongoing pregnancies under $\mathrm{LH}$ supplementation, in which the benefits appeared to be more evident for low responders [23]. Hence, we don't propose the long-acting FSH plus MPA regimen in low responders. Because of limited comprehension about the preventive effect of endogenous progesterone on premature LH surge in the past, previous investigation about luteal start utilizing pure $\mathrm{FSH}[3,24,25]$ usually initiated daily $\mathrm{GnRH}$ antagonist from the first day of ovarian stimulation. In these regimens both $\mathrm{GnRH}$ antagonists and endogenous progesterone exerted suppressive influence and consequently resulted in much more profound pituitary suppression and therefore much higher dosage of FSH or longer stimulation duration. Long-acting FSH has also been used in a fashion of random start with antagonist protocol [26], but there is lack of investigation specific to luteal stimulation. As for PPOS without GnRH antagonist, the group in 
China led by Kuang $[8,10,27]$ demonstrated that MPA leads to stronger pituitary suppression in PPOS as compared with utrogestan and dydrogesterone, under which the LH values gradually declined in the first 5 days of MPA co-treatment. The proportion of women with profound pituitary suppression, which is defined as serum LH less than $1.0 \mathrm{IU} / \mathrm{L}$ on the trigger day, was $32 \%$ in the PPOS with daily MPA $10 \mathrm{mg}$ commenced along with gonadotrophin stimulation [28]. The aforementioned researches used HMG for ovarian stimulation; therefore, whether PPOS without LH supplementation is adequate remains unclear. The only study utilizing urinary FSH to compare with two brands of HMG in PPOS for normal responders [29] showed no differences in the number of retrieved oocytes, mature oocytes as well as fertilization, cleavage, embryo quality, and pregnancy outcomes among groups, even under subanalysis upon patients with LH less than $0.68 \mathrm{IU} / \mathrm{L}$ on the trigger day. In our regimen, we arbitrarily started MPA supplement from the second day of gonadotrophin stimulation, and the LH levels on stimulation day 8 were measured to be $2.70 \pm 1.84 \mathrm{IU} / \mathrm{L}$ in our previous study [14] and $2.85 \pm 2.40 \mathrm{IU} / \mathrm{L}$ in the current investigation (data not shown). After stimulation day $8, \mathrm{HMG}$ was added until trigger criteria were met. We demonstrated that the reproductive outcomes of long-acting FSH + MPA are satisfactory and comparable to those of long-acting $\mathrm{FSH}+$ antagonist. Certainly, further randomized controlled trials are needed.

Different progestin gives rise to different level of pituitary suppression $[10,27]$. Previous researches showed dydrogesterone $20 \mathrm{mg} / \mathrm{d}$ has less pituitary suppression than MPA $10 \mathrm{mg} / \mathrm{d}$ but more than utrogestan $100 \mathrm{mg} / \mathrm{d}$. The pituitary suppression in utrogestan-PPOS is dose-dependent [30]; in contrast, MPA shows no difference in the proportion of profound pituitary suppression between $4 \mathrm{mg} / \mathrm{d}$ and $10 \mathrm{mg} / \mathrm{d}$ [28]. Despite various levels of pituitary suppression, there was no difference in the reproductive outcomes among various types and dosages of progestogen in the studies upon normal responders [10, $27,30]$. The difference might emerge in low responders stimulated with only FSH instead of HMG or FSH $+\mathrm{LH}$. On the other hand, it seems that clomiphene co-administration is able to avoid LH over-suppression in PPOS, which resulted in an initial slight rise followed by a downward trend in $\mathrm{LH}$, although the limited data came from normal responders [31] and high responders [32]. If we design to extend our extremely patient-friendly regimen to low responders, different progestin and clomiphene combinations may be considered in PPOS with long-acting FSH where no exogenous LH is given in the first week.

Almost all previous relevant studies unanimously required higher dosage of HMG with or without longer duration for stimulation in MPA-PPOS $[8,12,33]$. In our finding, there was no significant difference in stimulation duration and gonadotrophin consumption between PPOS and antagonist protocol. There are two possible explanations: first, the comparison protocol in all of the aforementioned PPOS studies in the literature was short protocol, which had flare-up effects that were absent in antagonist protocol used in our study; second, we started MPA from the second day of gonadotrophin stimulation, which was one day later than the PPOS protocol in previous studies mentioned above and might lessen the pituitary suppression. Another retrospective cohort study comparing PPOS versus flexible antagonist protocol in the same donor aged between 23 and 29 years old administered MPA $10 \mathrm{mg} /$ day from the 7th day of daily FSH stimulation or when the leading follicle reached $14 \mathrm{~mm}$, whichever came first, and named this method as flexible progestin primed ovarian stimulation (fPPOS). Stimulation duration, gonadotropin consumption, and duration of GnRH antagonist/MPA administration were similar, and no premature 
ovulation occurred in either group. There were significantly more metaphase II oocytes in fPPOS cycles than in GnRH antagonist cycles. Recipients of fresh oocytes from fPPOS and GnRH antagonist cycles had similar cleavage, implantation, and live birth/ongoing pregnancy rates [34]. As to PPOS versus long protocol which suppresses pituitary function much more than short and antagonist protocol, there was a retrospective self-controlled study conducted on 104 aged infertile patients who failed in their first IVF/ICSI attempt by means of long protocol. The oocyte utilization rate and good-quality embryo rate in PPOS cycles were significantly higher. The duration of stimulation, total dosage of gonadotrophin, the number of oocytes retrieved, and fertilization rate were similar between PPOS and long protocols [35].

According to previous investigation, the fertility potential of oocytes collected in the presence of exogenous progestogen, regardless of MPA [8, 12], dydrogesterone $[10,27,34]$, or utrogestan $[9,13,36]$, are as competent as those collected via conventional stimulation protocols. An interesting big data revealed no difference in the reproductive outcome of the oocytes induced in the presence or absence of levonorgestrel-releasing intrauterine device [37]. Despite highest concentration of levonorgestrel within the uterus, serum level of levonorgestrel varied between 134 and $191 \mathrm{pg} / \mathrm{ml}$ [38], thus offering further observation of the effect of progestin on ovarian stimulation outcomes. In regards to endogenous progesterone, the reproductive outcomes of oocytes stimulated during luteal phase have already been qualified on a large scale [5-7].

The LH suppressive effect of progestogen has been utilized in the development of oral contraceptives, but has not been applied in ovarian stimulation for ART until remarkable improvement in freezing/thawing outcomes brought about by vitrification. 'Freeze all' is mandatory in PPOS. The leading benefit about FET is to eliminate the risk of late-onset OHSS without compromising implantation rate [39]. There is no absolute predictor for OHSS and a presumed normal responder according to AMH or AFC still could have potential OHSS risk [40]. In our center, for that reason, freeze-all policy is applied for both normal and high responders, and the extremely patient-friendly protocol lightens their load in the physical/mental/economic aspects. To alleviate the stress from injections, there were some regimens described in the previous literature. Long-acting $\mathrm{GnRH}$ agonist depot was anticipated to be better than short-acting ones. In a retrospective study including more than four hundred patients, however, higher OHSS risk and inferior reproductive outcomes were noted in the long-acting group [41], albeit no significant difference in another meta-analysis [42]. For better or worse, daily shots of gonadotrophins were still needed in the aforementioned downregulation protocols. It was proposed that $\mathrm{GnRH}$ antagonist can be used occasionally only when serum LH exceeds $6 \mathrm{IU} / \mathrm{L}$ during ovarian stimulation started with long-acting FSH (corifollitropin alfa) [43]. In spite of reduced injections, this protocol needs frequent blood tests to monitor LH titers for timely initiation of antagonist injection, which is bothersome and stressful on the other hand. Despite close follow-up, moreover, it is still possible to miss some LH peaks. Without any prevention, the rate of premature LH surge during ovarian stimulation was $20-25 \%$ according to the previous literature [44]. Using our method, the first visit was arranged at one week after long-acting FSH injection, and only an average of 1.4 visits were needed before trigger, which is convenient and friendly for the patients. 


\section{Conclusion}

Our extremely patient-friendly protocol of long-acting FSH plus MPA, with an average of 3.5 shots and 1.4 visits before trigger, displayed satisfactory stimulation and reproductive outcomes that are comparable to those of antagonist protocol.

\section{List Of Abbreviations}

antral follicle count (AFC)

assisted reproductive technology (ART)

body mass index (BMI)

estradiol (E2)

frozen-thawed embryo transfer (FET)

follicular stimulation hormone (FSH), recombinant FSH (rFSH)

gonadotrophin-releasing hormone $(\mathrm{GnRH})$

human chorionic gonadotrophin (hCG)

human menopausal gonadotrophin (HMG)

intracytoplasmic sperm injection (ICSI)

in vitro fertilization (IVF)

luteinizing hormone (LH)

medroxyprogesterone acetate (MPA)

ovarian hyperstimulation syndrome (OHSS)

progesterone $(P)$

progestin-primed ovarian stimulation (PPOS)

standard deviation (SD)

subcutaneous (sc)

IU=international unit

$\mathrm{Kg}=$ kilogram 
L=liter

\section{Declarations}

\section{Acknowledgments}

The authors thank Dr. Shang-Liang Wu at Biostatistics Task Force and Dr. Chi-Mou Juang at Department of Obstetrics and Gynecology, Taipei Veterans General Hospital for their statistical advice.

\section{Authors' contributions}

Huang $\mathrm{CY}$ wrote the original draft.

Chen GY collected and analyzed the data.

Shieh ML was in charge of investigation.

Li HY was responsible for conceptualization and draft revision. All authors read and approved the final manuscript.

\section{Funding}

The study was partially funded by the Ministry of Science and Technology (MOST 106-2314-B-010-042) and Taipei Veterans General Hospital (V107C-107).

\section{Availability of data and materials}

The datasets used and/or analysed during the current study are available from the corresponding author on reasonable request.

\section{Ethics approval}

Ethical approval for this study was obtained from the Institutional Review Board of Taipei Veterans General Hospital (2018-12-005BC)

\section{Consent for publication}

Not applicable

\section{Competing interests}

Huang CY has no competing interests to declare.

Chen GY has no competing interests to declare.

Shieh ML has no competing interests to declare. 
Li HY has no competing interests to declare.

\section{References}

1. Maheshwari A, Gibreel A, Siristatidis CS, Bhattacharya S. Gonadotrophin releasing hormone agonist protocols for pituitary suppression in assisted reproduction. Cochrane Database Syst Rev. 2011: CD006919. doi: 10.1002/14651858.CD006919.pub3

2. Al-Inany HG, Youssef MA, Ayeleke RO, Brown J, Lam WS, Broekmans FJ. Gonadotrophin-releasing hormone antagonists for assisted reproductive technology. Cochrane Database Syst Rev. 2016:CD001750. doi: 10.1002/14651858.CD001750.pub4.

3. von Wolff M, Thaler CJ, Frambach T, Zeeb C, Lawrenz B, Popovici RM, Strowitzki T. Ovarian stimulation to cryopreserve fertilized oocytes in cancer patients can be started in the luteal phase. Fertil Steril. 2009;92(4):1360-5. doi: 10.1016/j.fertnstert.2008.08.011. Epub 2008 Oct 18.

4. Boots $C E$, Meister M, Cooper AR, Hardi A, Jungheim ES. Ovarian stimulation in the luteal phase: systematic review and meta-analysis. J Assist Reprod Genet. 2016;33(8):971-80. doi: 10.1007/s10815-016-0721-5. Epub 2016 May 4.

5. Kuang Y, Hong Q, Chen Q, Lyu Q, Ai A, Fu Y, Shoham Z. Luteal-phase ovarian stimulation is feasible for producing competent oocytes in women undergoing in vitro fertilization/intracytoplasmic sperm injection treatment, with optimal pregnancy outcomes in frozen-thawed embryo transfer cycles. Fertil Steril. 2014;101(1):105-11. doi: 10.1016/j.fertnstert.2013.09.007. Epub 2013 Oct 23.

6. Wang N, Wang Y, Chen Q, Dong J, Tian H, Fu Y, et al. Luteal-phase ovarian stimulation vs conventional ovarian stimulation in patients with normal ovarian reserve treated for IVF: a large retrospective cohort study. Clin Endocrinol (Oxf). 2016;84(5):720-8. doi: 10.1111/cen.12983. Epub 2015 Dec 21.

7. Chen H, Wang Y, Lyu Q, Ai A, Fu Y, Tian H, et al. Comparison of live-birth defects after luteal-phase ovarian stimulation vs. conventional ovarian stimulation for in vitro fertilization and vitrified embryo transfer cycles. Fertil Steril. 2015;103(5):1194-1201.e2. doi: 10.1016/j.fertnstert.2015.02.020. Epub 2015 Mar 23.

8. Kuang Y, Chen Q, Fu Y, Wang Y, Hong Q, Lyu Q, et al. Medroxyprogesterone acetate is an effective oral alternative for preventing premature luteinizing hormone surges in women undergoing controlled ovarian hyperstimulation for in vitro fertilization. Fertil Steril. 2015;104(1):62-70. e3. doi: 10.1016/j.fertnstert.2015.03.022. Epub 2015 May 5.

9. Zhu X, Zhang X, Fu Y. Utrogestan as an effective oral alternative for preventing premature luteinizing hormone surges in women undergoing controlled ovarian hyperstimulation for in vitro fertilization. Medicine (Baltimore). 2015;94(21):e909. doi: 10.1097/MD.0000000000000909.

10. Zhu X, Ye H, Fu Y. Duphaston and human menopausal gonadotropin protocol in normally ovulatory women undergoing controlled ovarian hyperstimulation during in vitro fertilization/intracytoplasmic sperm injection treatments in combination with embryo cryopreservation. Fertil Steril 2017;108(3):505-512.e2. doi: 10.1016/j.fertnstert.2017.06.017. Epub 2017 Jul 8. 
11. Massin N. New stimulation regimens: endogenous and exogenous progesterone use to block the LH surge during ovarian stimulation for IVF. Hum Reprod Update. 2017;23(2):211-20. doi: 10.1093/humupd/dmw047.

12. Wang $Y$, Chen $\mathrm{Q}$, Wang N, Chen H, Lyu Q, Kuang Y. Controlled ovarian stimulation using Medroxyprogesterone acetate and hMG in patients with polycystic ovary syndrome treated for IVF: a double-blind randomized crossover clinical trial. Medicine (Baltimore). 2016;95(9):e2939. doi: 10.1097/MD.0000000000002939.

13. Zhu X, Ye H, Fu Y. The Utrogestan and hMG protocol in patients with polycystic ovarian syndrome undergoing controlled ovarian hyperstimulation during IVF/ICSI treatments. Medicine (Baltimore) 2016;95:e4193. doi: 10.1097/MD.0000000000004193.

14. Huang $C Y$, Chen GY, Shieh ML, Li HY. An extremely patient-friendly and efficient stimulation protocol for assisted reproductive technology in normal and high responders. Reprod Biol Endocrinol. 2018;16(1):18. doi: 10.1186/s12958-018-0335-0.

15. Tobler KJ, Zhao Y, Weissman A, Majumdar A, Leong M, Shoham Z. Worldwide survey of IVF practices: trigger, retrieval and embryo transfer techniques. Arch Gynecol Obstet. 2014;290(3):561-8. doi: 10.1007/s00404-014-3232-6. Epub 2014 Apr 18.

16. de Greef R, Zandvliet AS, de Haan AF, ljzerman-Boon PC, Marintcheva-Petrova M, Mannaerts BM. Dose selection of corifollitropin alfa by modeling and simulation in controlled ovarian stimulation. Clin Pharmacol Ther. 2010;88(1): 79-87. doi: 10.1038/clpt.2010.54. Epub 2010 Jun 2. Erratum in: Clin Pharmacol Ther. 2010;88(3):424.

17. Veeck LL. An atlas of human gametes and conceptuses. New York: Parthenon; 1998. p. 40-5.

18. Fares FA, Suganuma N, Nishimori K, LaPolt PS, Hsueh AJ, Boime I. Design of a long-acting follitropin agonist by fusing the $\mathrm{C}$-terminal sequence of the chorionic gonadotropin beta subunit to the follitropin beta subunit. Proc Natl Acad Sci U S A. 1992;89(10):4304-8.

19. Fauser BC, Mannaerts BM, Devroey P, Leader A, Boime I, Baird DT. Advances in recombinant DNA technology: corifollitropin alfa, a hybrid molecule with sustained follicle-stimulating activity and reduced injection frequency. Hum Reprod Update. 2009;15(3):309-21. doi: 10.1093/humupd/dmn065. Epub 2009 Jan 30.

20. Pouwer AW, Farquhar C, Kremer JA. Long-acting FSH versus daily FSH for women undergoing assisted reproduction. Cochrane Database Syst Rev. 2015: CD009577. doi: 10.1002/14651858.CD009577.pub3. Review.

21. Hill MJ, Levens ED, Levy G, Ryan ME, Csokmay JM, DeCherney AH, Whitcomb BW. The use of recombinant luteinizing hormone in patients undergoing assisted reproductive techniques with advanced reproductive age: a systematic review and meta-analysis. Fertil Steril. 2012;97(5):110814. e1. doi: 10.1016/j.fertnstert.2012.01.130. Epub 2012 Feb 24.

22. Xiong Y, Bu Z, Dai W, Zhang M, Bao X, Sun Y. Recombinant luteinizing hormone supplementation in women undergoing in vitro fertilization/ intracytoplasmic sperm injection with gonadotropin 
releasing hormone antagonist protocol: a systematic review and meta-analysis. Reprod Biol Endocrinol. 2014;12:109. doi: 10.1186/1477-7827-12-109.

23. Mochtar MH, Danhof NA, Ayeleke RO, Van der Veen F, van Wely M. Recombinant luteinizing hormone $(\mathrm{rLH})$ and recombinant follicle stimulating hormone $(\mathrm{rFSH})$ for ovarian stimulation in IVF/ICSI cycles. Cochrane Database Syst Rev. 2017:CD005070. doi: 10.1002/14651858.CD005070.pub3. Review.

24. Buendgen NK, Schultze-Mosgau A, Cordes T, Diedrich K, Griesinger G. Initiation of ovarian stimulation independent of the menstrual cycle: a case-control study. Arch Gynecol Obstet. 2013;288(4):901-4. doi: 10.1007/s00404-013-2794-z. Epub 2013 Apr 3.

25. Martínez F, Clua E, Devesa M, Rodríguez I, Arroyo G, González C, et al. Comparison of starting ovarian stimulation on day 2 versus day 15 of the menstrual cycle in the same oocyte donor and pregnancy rates among the corresponding recipients of vitrified oocytes. Fertil Steril. 2014; 102(5):1307-11. doi: 10.1016/j.fertnstert.2014.07.741. Epub 2014 Aug 22.

26. Sarais V, Paffoni A, Pagliardini L, Filippi F, Martinelli F, Mangili G, et al. Long-acting recombinant folliclestimulating hormone in random-start ovarian stimulation protocols for fertility preservation in women with cancer. Acta Obstet Gynecol Scand 2017;96(8):949-953. doi: 10.1111/aogs.13146. Epub 2017 May 16.

27. Yu S, Long H, Chang HY, Liu Y, Gao H, Zhu J, et al. New application of dydrogesterone as a part of a progestin-primed ovarian stimulation protocol for IVF: a randomized controlled trial including 516 first IVF/ICSI cycles.

Hum Reprod. 2018;33(2):229-237. doi: 10.1093/humrep/dex367.

28. Dong J, Wang Y, Chai WR, Hong QQ, Wang NL, Sun LH, et al. The pregnancy outcome of progestinprimed ovarian stimulation using 4 versus $10 \mathrm{mg}$ of medroxyprogesterone acetate per day in infertile women undergoing in vitro fertilisation: a randomised controlled trial. BJOG. 2017;124(7):1048-55. doi: 10.1111/1471-0528.14622.

29. Zhu X, Ye J, Fu Y, Ai A, Cai R, Wang Y, et al. The effect of human chorionic gonadotrophin contained in human menopausal gonadotropin on the clinical outcomes during progestin-primed ovarian stimulation. Oncotarget. 2017;8(50):87340-87352. doi: 10.18632/oncotarget.20508. eCollection 2017 Oct 20.

30. Zhu X, Ye H, Fu Y. Use of Utrogestan during controlled ovarian hyperstimulation in normally ovulating women undergoing in vitro fertilization or intracytoplasmic sperm injection treatments in combination with a "freeze all" strategy: a randomized controlled dose-finding study of $100 \mathrm{mg}$ versus 200 mg. Fertil Steril. 2017;107(2):379-386.e4. doi: 10.1016/j.fertnstert.2016.10.030. Epub 2016 Nov 16.

31. Liu Y, Chen Q, Yu S, Wang Y, He W, Chang HY, et al. Progestin-primed ovarian stimulation with or without clomiphene citrate supplementation in normal ovulatory women undergoing in vitro fertilization/intracytoplasmic sperm injection: A prospective randomized controlled trial. Clin Endocrinol (Oxf). 2018;88(3):442-452. doi: 10.1111/cen.13532. Epub 2018 Jan 4. 
32. Ye H, Tian H, He W, Lyu Q, Kuang Y, Chen Q, Sun L. Progestin-primed milder stimulation with clomiphene citrate yields fewer oocytes and suboptimal pregnancy outcomes compared with the standard progestin-primed ovarian stimulation in infertile women with polycystic ovarian syndrome. Reprod Biol Endocrinol. 2018;16(1):53. doi: 10.1186/s12958-018-0373-7.

33. Guo H, Wang Y, Chen Q, Chai W, Sun L, Ai A, et al. Use of medroxyprogesterone acetate in women with ovarian endometriosis undergoing controlled ovarian hyperstimulation for in vitro fertilization. Sci Rep. 2017;7(1):11927. doi: 10.1038/s41598-017-12151-7.

34. Yildiz S, Turkgeldi E, Angun B, Eraslan A, Urman B, Ata B. Comparison of a novel flexible progestin primed ovarian stimulation protocol and the flexible gonadotropin-releasing hormone antagonist protocol for assisted reproductive technology. Fertil Steril. 2019;112(4):677-683. doi: 10.1016/j.fertnstert.2019.06.009. Epub 2019 Jul 29.

35. Chen YM, Qi QR, Xie QZ, Yang YF, Xia Y, Zhou XD. Effect of Progestin-primed Ovarian Stimulation Protocol on Outcomes of Aged Infertile Women Who Failed to Get Pregnant in the First IVF/ ICSI Cycle: A Self-controlled Study. Curr Med Sci. 2018;38(3):513-518. doi: 10.1007/s11596-018-1908-z. Epub 2018 Jun 22.

36. Zhu X, Ye H, Fu Y. Comparison of neonatal outcomes following progesterone use during ovarian stimulation with frozen-thawed embryo transfer. Sci Rep. 2017;7(1):7835. doi: 10.1038/s41598-01708472-2.

37. Adeleye AJ, Aghajanova L, Kao CN, Cedars MI, Sauer MV. Impact of the levonorgestrel-releasing intrauterine device on controlled ovarian stimulation outcomes. Fertil Steril. 2018;110(1):83-88. doi: 10.1016/j.fertnstert.2018.03.022. Epub 2018 Jun 6.

38. Seeber B, Ziehr SC, Gschließer A, Moser C, Mattle V, Seger C, et al. Quantitative levonorgestrel plasma level measurements in patients with regular and prolonged use of the levonorgestrel-releasing intrauterine system. Contraception 2012;86(4):345-9. doi: 10.1016/j.contraception.2012.01.015. Epub 2012 Mar 6. Erratum in: Contraception. 2013;88(1):194.

39. Blockeel C, Drakopoulos P, Santos-Ribeiro S, Polyzos NP, Tournaye H. A fresh look at the freeze-all protocol: a SWOT analysis. Hum Reprod. 2016;31(3): 491-7. doi: 10.1093/humrep/dev339. Epub 2016 Jan 2.

40. Humaidan P, Quartarolo J, Papanikolaou EG. Preventing ovarian hyperstimulation syndrome: guidance for the clinician. Fertil Steril. 2010;94(2):389-400. doi: 10.1016/j.fertnstert.2010.03.028. Epub 2010 Apr 22.

41. Duan L, Bao S, Li K, Teng X, Hong L, Zhao X. Comparing the long-acting and short-acting forms of gonadotropin-releasing hormone agonists in the long protocol of IVF/ICSI cycles: a retrospective study. J Obstet Gynaecol Res. 2017;43(6):1037-42. doi: 10.1111/jog.13305. Epub 2017 Apr 19.

42. Albuquerque LE, Tso LO, Saconato H, Albuquerque MC, Macedo CR. Depot versus daily administration of gonadotrophin-releasing hormone agonist protocols for pituitary down regulation in assisted reproduction cycles. Cochrane Database Syst Rev. 2013:CD002808. doi: 10.1002/14651858.CD002808.pub3. 
43. Wang HL, Lai HH, Chuang TH, Shih YW, Huang SC, Lee MJ, Chen SU. A patient friendly Corifollitropin alfa protocol without routine pituitary suppression in normal responders. PLoS One. 2016;11(4):e0154123. doi: 10.1371/journal.pone.0154123. eCollection 2016.

44. Eibschitz I, Belaisch-Allart JC, Frydman R. In vitro fertilization management and results in stimulated cycles with spontaneous luteinizing hormone discharge. Fertil Steril. 1986;45(2):231-6.

\section{Figures}


(1)

Long-acting FSH

HMG Trigger

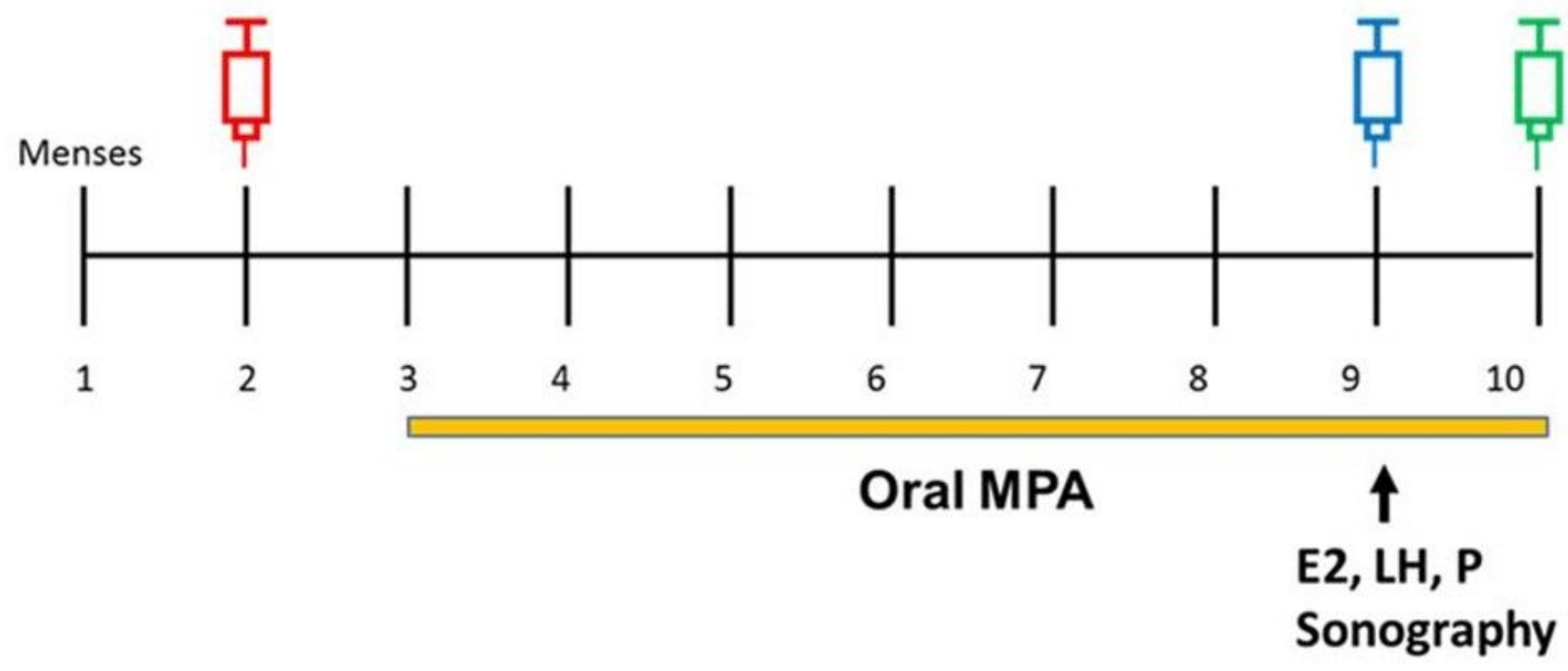

(2)

Long-acting FSH

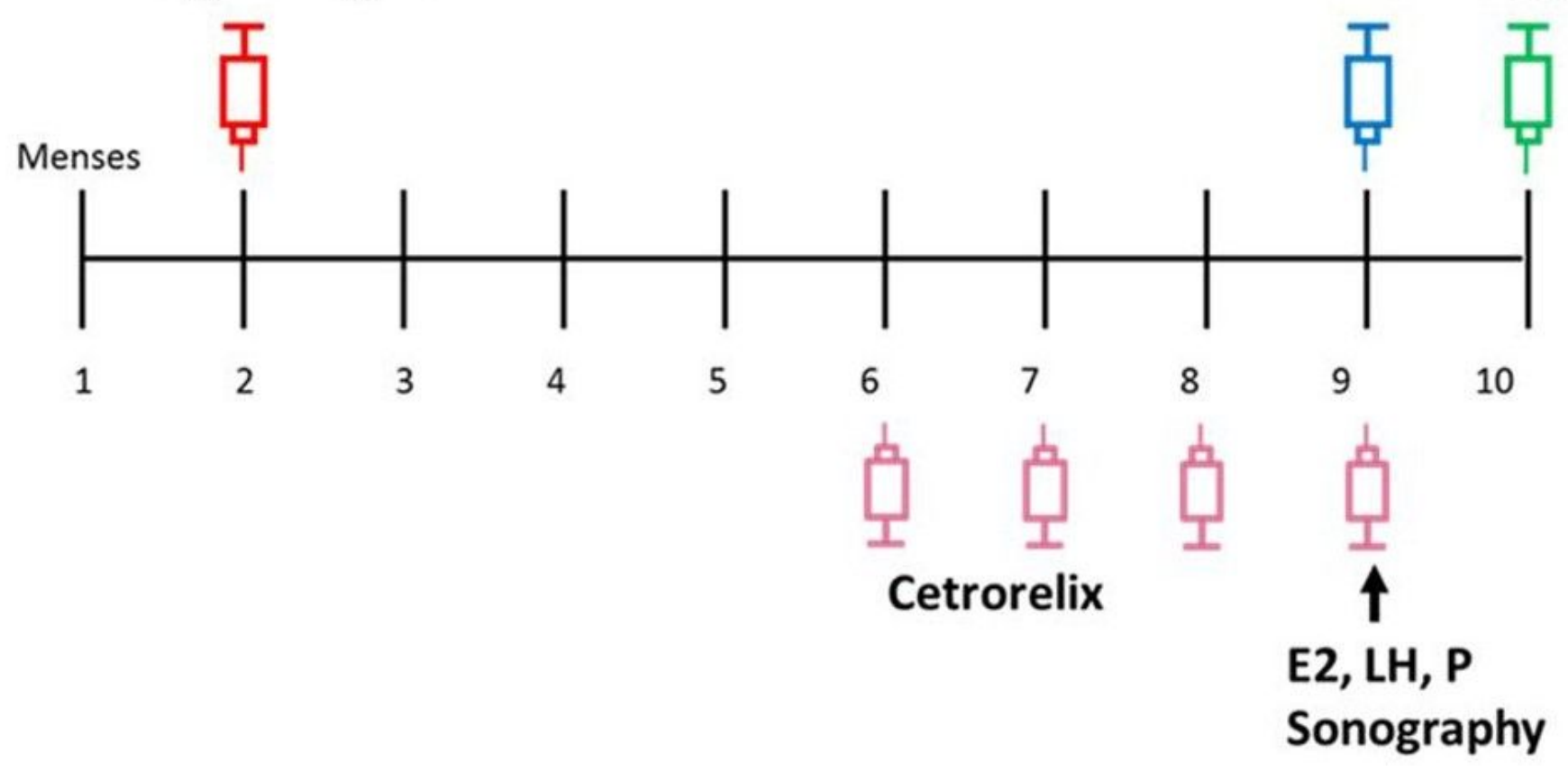

Figure 1

Simplified schematic descriptions of the two treatment protocols. Ovarian stimulation began with longacting FSH (corifollitropin alfa) at early follicular phase. In cycles with PPOS [Fig. 1-(1), group 1], patients took medroxyprogesterone acetate (MPA) $5 \mathrm{mg}$ twice a day from the day after long-acting FSH injection. In cycles with antagonist protocol [Fig. 1-(2), group 2], daily cetrorelix $0.25 \mathrm{mg}$ sc was initiated from the evening of stimulation day 5 . Seven days after long-acting FSH injection, the follicle development was 
monitored by transvaginal sonography as well as serum E2, LH and P measurements. As long as at least three leading follicles reached above $17 \mathrm{~mm}$, patients were triggered at night. If the trigger criterion was not met, additional HMG 150-225 IU/day would be administered for days depending on the prediction according to the measurement on stimulation day 8 . If needed, additional follicle monitoring would be performed every 2-3 days before trigger. Patients in group 1 took the final tablet of MPA in the morning of the trigger day, whereas patients in group 2 received the final cetrorelix in the evening before the trigger day.

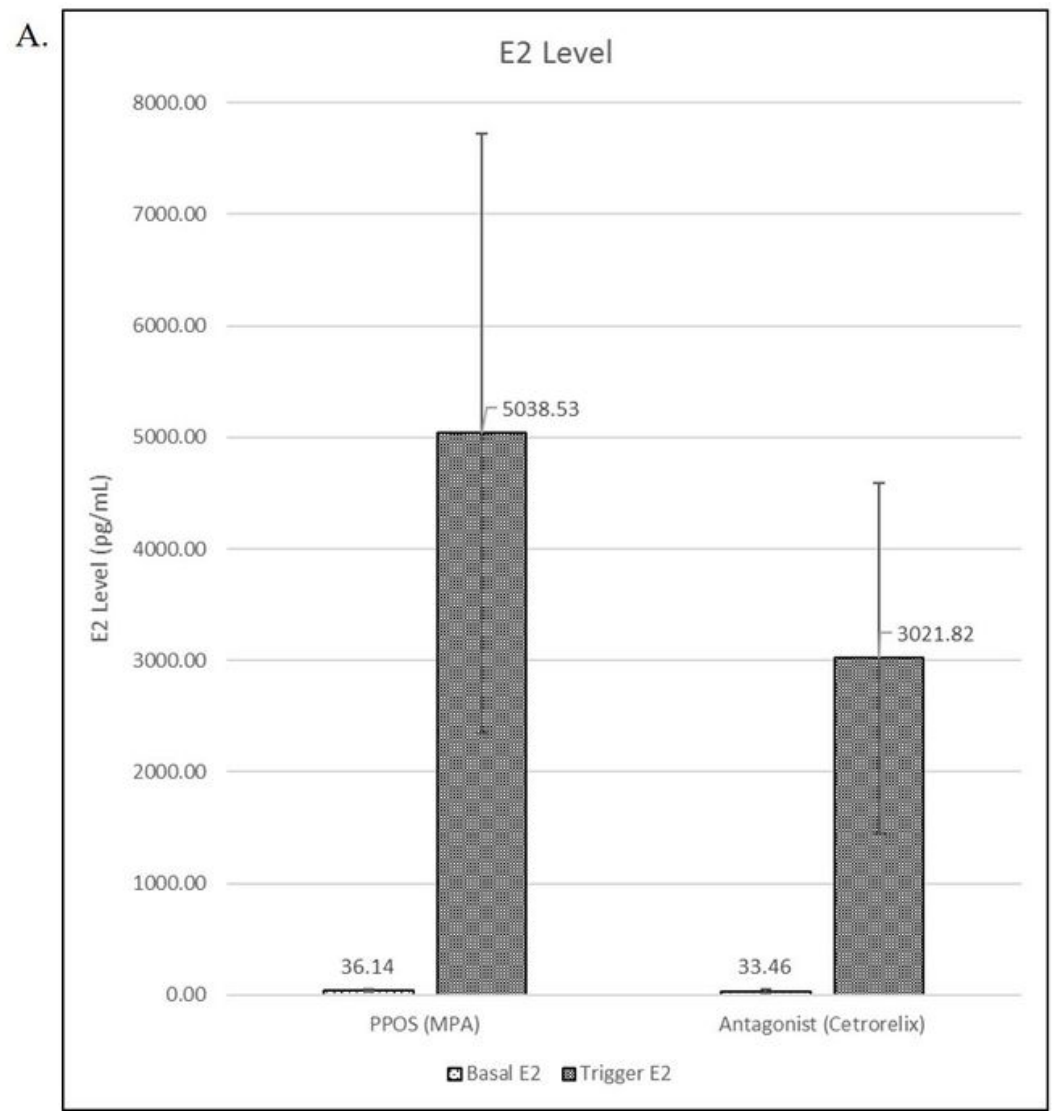

B.

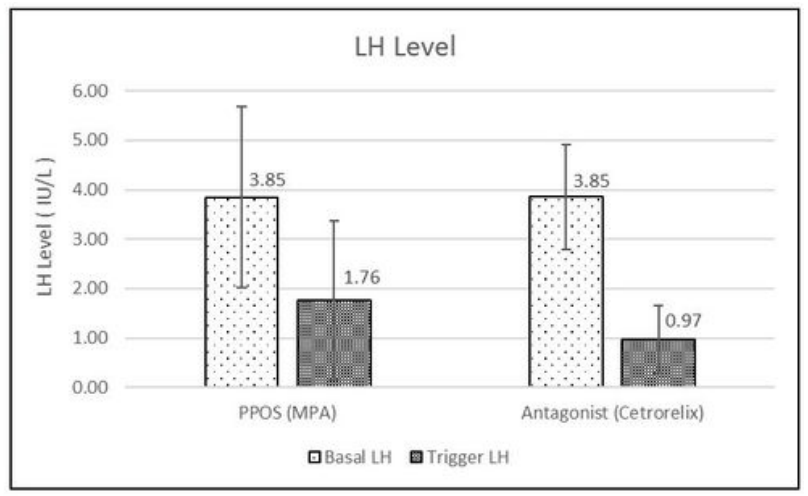

C.

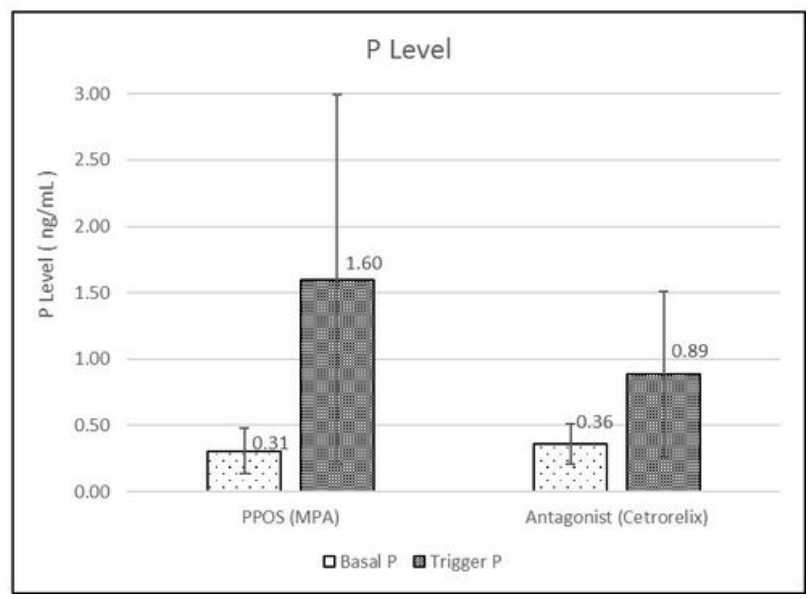

\section{Figure 2}

Hormonal changes in patients undergoing PPOS (group 1) versus antagonist protocol (group 2) A. Serum E2 changes from basal status to trigger day B. Serum LH changes from basal status to trigger day C. Serum $\mathrm{P}$ changes from basal status to trigger day 